\title{
Microcavity Lasers on Polymer Materials: Boundary Integral Equation Modeling and Experiments
}

\author{
A. I. Nosich, ${ }^{1}$ E. I. Smotrova, ${ }^{1}$ M. Lebental, ${ }^{2}$ I. O. Sukharevsky, ${ }^{3}$ and A. Altintas ${ }^{3}$ \\ ${ }^{1}$ Laboratory of Micro and Nano Optics, Institute of Radio-Physics and Electronics NASU, Kharkiv, Ukraine \\ ${ }^{2}$ Laboratory of Quantum and Molecular Photonics, ENS de Cachan, Cachan, France \\ ${ }^{3}$ Department of Electrical and Electronic Engineering, Bilkent University, Ankara, Turkey
}

\begin{abstract}
We consider the modeling and experiments with polymer dye-doped lasers shaped as thin flat cavities, allowing one to consider them as two-dimensional (2-D) active cavities. We focus our modeling on the H-polarized electromagnetic field in a kite-shaped laser. Assuming that the lasing-mode frequency is real-valued, we look for it together with the corresponding threshold value of material gain. Such electromagnetic-field problem is reduced to the Muller set of the boundary integral equations (MBIE), discretization of which yields determinantal equation. Numerical results reveal various types of modes existing in the kite including the perturbed whispering gallery (WG) modes that have the lowest thresholds. Their far-field emission patterns show good agreement with the measurements.
\end{abstract}

Keywords - microcavity laser; threshold; integral equationsr

\section{INTRODUCTION}

Since the first experiments in the early 1990s, it is known that lasers with microcavities shaped as thin flat circular disks with smooth enough rims can sustain whisperinggallery modes with very low thresholds $[1,2]$. Such lasers can be crystalline doped with erbium, semiconductor or dyedoped polymer ones. They can stand on pedestals or lay on less-optically-dense substrates. If the thickness of a flat cavity is smaller than the optical wavelength, then such microcavity can be studied in the 2-D approximation, where the bulk refractive index is replaced with its effective value. Then the modeling predicts that the WG mode thresholds are exponentially small relatively to the in-plane optical size of ideally circular cavity [3] although in practice they are restricted by the surface roughness. It is noteworthy that each mode with azimuth index $m>0$ is double degenerate in a circular cavity. The emission on these modes is predominantly in the cavity plane where it has low directivity because of $2 m$ identical beams [1-3]. This is a serious drawback because photonic applications often require emitters with larger directivity.

The review papers [4-6] contain references to the studies on the modes of the non-circular cavities, which can provide better directionality. This included fully convex contours like ellipse, stadium, cut circle, various regular polygons, and others, and also partially concave "notched" contours" [716]. Particularly high expectations had been associated with a resonator having in-plane contour given by an Archimedean spiral with a small step [17-20].

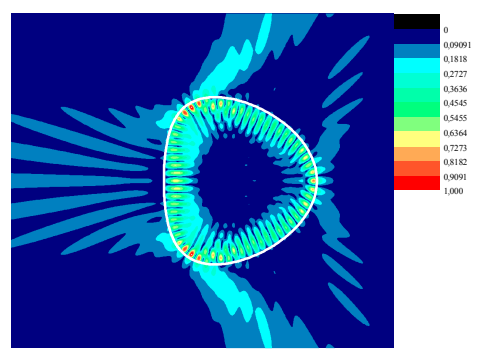

(a)

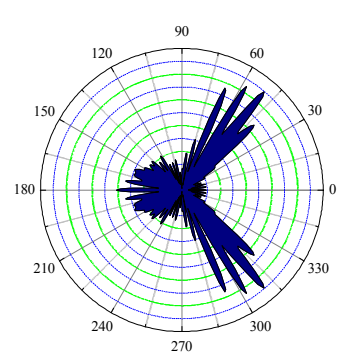

(b)

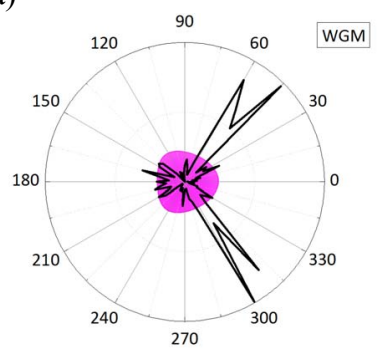

(c)

Fig. 1. 2-D microcavity laser shaped as a kite with refractive index $\alpha=1.5$ and deformation parameter $\delta=0.165$, filled in with an active material. Near (a) and far (b) field patterns computed at $k a=23.6404$ and $\gamma=9.7644 * 10^{-3}$; far filed measured (c) for $a=70 \mu \mathrm{m}$ at $\lambda=600 \mathrm{~nm}$.

Still the thresholds of lasing of a spiral laser were rather high because of strongly perturbing nature of the wavelengthsize step directly on the contour. Therefore the research gradually shifted to smoother shapes, for instance provided by the limacon curve [21-23]. One of the other perturbations of the circle is given by a "kite" curve. Unlike limacon, which obtains an inflection point if the perturbation is above a certain value, a kite is always smooth although it can be both fully and partially convex depending on the deformation parameter.

It is necessary to emphasize that simulations of microlaser modes in the past were performed within the passive cavity model where instead of the actual thresholds one looked for the mode quality factors. As demonstrated in [24], low threshold is not equivalent to high Q-factor although the former is inverse proportional to the latter - the overlap between the active region and the modal electric field is equally important. The adequate linear modeling of lasers is provided by the Lasing Eigenvalue Problem (LEP) specifically tailored to consider open resonators equipped with active regions $[3,5,24,25]$. LEP implies introduction of material gain 


\section{IEEE 35th International Conference on Electronics and Nanotechnology (ELNANO)}

into the whole microcavity or at least an active region and enables extraction of the lasing frequencies and associated threshold values of material gain as eigenvalues.

\section{LASING EIGENVALUE PROBLEM}

In Fig. 1, we show the geometry of the considered 2-D microcavity laser. We suppose that the cavity shape is given by the equation,

$$
x(t)=a(\cos t+\delta \cos 2 t-\delta), \quad y(t)=a \sin t, \quad 0 \leq t \leq 2 \pi,(1)
$$

which turns into the circle of the radius $a$ if the deformation parameter $\delta=0$. This cavity is filled with an active material characterized with a complex-valued refractive index $v=\alpha-i \gamma$, where $\alpha>0$ is the effective refractive index and $\gamma>0$ is the bulk material gain. The host medium is vacuum. The electromagnetic field is assumed to depend on the time as $e^{-i \omega t}$; we will denote the free-space wavenumber as $k=\omega / c=2 \pi / \lambda$, where $\lambda$ is the wavelength and $c$ is the light velocity. The function, which we are looking for is the magnetic field $H_{z}$ component. It must satisfy the Helmholtz equation with the corresponding wavenumber inside each material, the Sommerfeld (outgoing-wave) radiation condition at infinity, the condition of local power boundedness, and the continuity of the tangential field components at the boundary. Guided by $[3,5,24,25]$, we considered the field problem as an eigenvalue problem and looked for the ordered pairs of realvalued numbers $(k a, \gamma)$. In each pair, the first number is the lasing mode normalized frequency and the second is the threshold value of gain necessary to make $k a$ real, i.e. to achieve the lasing. Note that the gain per unit length is $g=k \gamma$.

\section{NUMERICAL STUDY OF THE LASING MODES}

The full-wave numerical analysis of the wavelength-scale dielectric resonators are normally associated with the volume and boundary IEs [26-31]. The latter equations are usually more attractive as they demand, in 2-D, the discretization of only the scatterer contour instead of its area.

However, one should keep in mind that many forms of the boundary IEs are contaminated by the presence of spurious eigenvalues [32,33]. For instance, all the works [7,9-12,18,19] dealt with such IE although this fact was fully acknowledged only in the paper [10]. The spurious eigenvalues are the real frequencies (zero-threshold values, in LEP) that are the eigenvalues of the interior electromagnetic problem where the boundary is assumed perfectly electrically conducting and the inside filling is free space. They have no physical meaning. Their presence spoils the computation of low-threshold modes because iterative-search codes easily jump to the nearby false roots of the corresponding determinantal equations.

Note that the analytical regularization of such defective IEs, i.e. their reduction to the Fredholm second-kind matrix equations, achieved, for instance, using their projection to specially tailored Galerkin basis functions [30] does not remove the defect mentioned. As discussed in [25], the spurious eigenvalues are absent for the MBIE [34], which, in the 2-D case, is a pair of coupled second-kind IEs for the field components tangential to the scatterer contour. This is unlike the combined-field IE, which only pushes the spurious eigenvalues off from the real axis. As the kernels of MBIE are smooth or integrable functions [25], the Fredholm theorems guarantee the convergence of conventional discretizations. In the past, MBIE were used in [35] together with the meshing of the contour and the application of the local basis functions. In [36], MBIE was alternatively projected to the trigonometric polynomials as global basis functions; in this case additional merits were provided by their regularizing properties with respect to MBIE kernels.

As a valuable alternative, the Nystrom-type meshless discretization, which exploits interpolation polynomials and quadrature formulas, also guarantees the convergence $[37,38]$. Here, we understand the convergence in mathematical sense, as ability to minimize the error of computations, to machine precision, by using progressively larger orders of discretization. In the this paper, we used the $2 \pi$-periodic parameterization [25], split all kernels of MBIE into logarithmic and smooth parts and used two different quadrature formulas for replacing the integrals with finite sums. After that we obtained determinantal equation for the LEP eigenvalues. We solved this equation using the iterative Newton method, which needed initial-guess values found from the visualization of the determinant relief on the plane $(k a, \gamma)$. Another approach can exploit the segmentation of the contour to a minimum number of regular parts and further use the numerical algorithm explained and validated in [39].

\section{EXPERIMENTS WITH POLYMER LASERS}

Experiments were performed on kite-shaped dye-doped polymer microlasers [17,40,41]. Planar dimensions of such microlasers were from 100 to $200 \mu \mathrm{m}$ that was several orders of magnitude larger than both the cavity thickness of $0.5 \mu \mathrm{m}$ and the emission wavelength of $0.6 \mu \mathrm{m}$. The fabrication workflow consisted of two steps. At first the dye-doped polymer layer was obtained by spin-coating a mixture of poly(methylmetacrylate) (Microchem 495 PMMA A6) polymer with $5 \mathrm{wt} \%$ of a high-purity non-commercial dye MD7 (4,4-difluoro-8-mesityl-3,5-di(naphthalen-1-yl)-4-bora$3 \mathrm{a}, 4 \mathrm{a}$-diaza-s-indacene) on a commercial $\mathrm{SiO} 2(2 \mu \mathrm{m}) / \mathrm{Si}$ substrate. The kite contour was then obtained in the doped PMMA layer by means of electron-beam lithography. This allowed achieving almost defect-free cavity surface with flat sidewalls. During experiment, a single microlaser was uniformly pumped from the top with a frequency doubled pulsed Nd:YVO $\mathrm{YV}_{4}$ laser $(532 \mathrm{~nm}, 500 \mathrm{ps}, 10 \mathrm{~Hz})$. The microlaser emission was then collected within the cavity plane using a lens with collection angle of $6^{\circ}$, which focused the emission into an optical fiber connected to a spectrometer equipped with a cooled CCD camera. All experiments have been carried out at room atmosphere and temperature.

The experimentally measured spectra had confirmed the coexistence of different types of modes, including the perturbed WG modes. The comparison of the far-field emission patterns is shown in Fig. 1. It demonstrates quite good agreement between the measurements and modelling 


\section{IEEE 35th International Conference on Electronics and Nanotechnology (ELNANO)}

despite the fact that the experimental cavity was much larger than the computed.

\section{CONCLUSIONS}

We presented results of accurate modeling of the lasing spectra and material gain thresholds for a 2-D kite-shaped microcavity laser. In such a laser, WG-like modes display the lowest thresholds if the deformation is small or moderate. The directionality of their emission is much higher than for a circular disk laser. The experiments done with dye-doped polymer laser of the same shape showed good agreement of the predicted and measured emission patterns.

\section{ACKNOWLEDGMENT}

This work was supported in part by the European Science Foundation via Research and Training Networks "Newfocus" and "Polatom."

\section{REFERENCES}

[1] S. L. McCall, A. F.J. Levi, R. E. Slusher, et al., "Whispering-gallery mode microdisk lasers," Appl. Phys. Lett., vol. 60, no 3, pp. 289-29, 1992.

[2] A.B. Matsko and V.S. Ilchenko, "Optical resonators with whispering-gallery modes, Pt. 1, Basics and Pt. 2, Applications," IEEE J. Sel. Top. Quant. Electron., vol. 12, no 1, pp. 3-32, 2006.

[3] E. I. Smotrova, A. I. Nosich, T. M. Benson, and P. Sewell, "Cold-cavity thresholds of microdisks with uniform and nonuniform gain: quasi-3-D modeling with accurate 2-D analysis," IEEE J. Sel. Top. Quant. Electron., vol. 11, no 5, pp. 1135-1142, 2005.

[4] H.G.L. Schwefel, et al., "Progress in asymmetric resonant cavities: using shape as a design parameter in dielectric microcavity lasers," In: Optical Microcavities, Ed. K. Vahala, World Scientific, Singapore, pp. 415-496, 2004.

[5] A.I. Nosich, et al., "Trends in microdisk laser research and linear optical modeling," Opt. Quant. Electron., vol. 39, no 15, pp. 1253-1272, 2007.

[6] T. Harayama and S. Shinohara, "Two-dimensional microcavity lasers," Laser Photonics Rev., vol. 5, no 2, pp. 247-281, 2011.

[7] J.-W. Ryu and M. Hentschel, "Designing coupled microcavity lasers for high-Q modes with unidirectional light emission," Opt. Lett., vol. 36, no 7, pp. 11161118, 2011.

[8] A.F.J. Levi, R.E. Slusher, S.L. McCall, et al., "Directional light coupling from microdisk lasers," Appl. Phys. Lett.m, vol. 62, no 6, pp. 561-563, 1993.

[9] H.G.L. Schwefel, N.B. Rex, H. E. Tureci, R. K. Chang, A.D. Stone, "Dramatic shape sensitivity of directional emission patterns from similarly deformed cylindrical polymer lasers," J. Opt. Soc. Am. B, vol. 21, no 5, pp. 923-934 2004.

[10] J. Wiersig, "Hexagonal dielectric resonators and microcrystal lasers," Phys. Rev. A, vol. 67, pp. 023807, 2003.

[11] S.-K. Kim, et al., "Highly directional emission from few-micron-size elliptical microdisks," Appl. Phys. Lett., vol. 84, no 6, pp. 861-863, 2004.

[12] S R. Dubertrand, et al., "Circular dielectric cavity and its deformations," Phys. Rev. A., vol. 77, no 1, pp. 013804(16), 2008.

[13] S.V. Boriskina, et al., "Q-factor and emission pattern control of the whispering gallery modes in notched microdisk resonators," IEEE J. Selected Topics Quant. Electron., vol. 12, no 1, pp. 66-70 2006.

[14] C. Lafargue, M. Lebental, A. Grigis, et al., "Localized lasing modes of triangular organic microlasers," Phys. Rev. E., vol. 90, no 5, pp. 052922/17, 2014.

[15] Q.J. Wang, C. Yan, N. Yu, et al., "Whispering-gallery mode resonators for highly unidirectional laser action," Proc. Nat. Ac. Sci., vol. 107, no 52, pp. 22407-22412, 2010.

[16] F. Courvoisier, V. Boutou, J. P. Wolf, R. K. Chang, and J. Zyss, "Deciphering output coupling mechanisms in spiral microcavities with femtosecond light bullets," Opt. Lett., vol. 30, no 7, pp. 737-740, 2005.
[17] T. Ben-Massaoud and J. Zyss, "Unidirectional laser emission from polymerbased spiral microdisks," Appl. Phys. Lett., vol. 86, pp. 241110, 2005.

[18] J. Wiersig and M. Hentschel, "Asymmetric scattering and nonorthogonal mode patterns in optical microspirals", Phys. Rev. A, vol. 73, pp. 031802, 2006.

[19] M. Hentschel, et al., "Angular emission characteristics of quantum cascade spiral microlasers," Opt. Expr., vol. 17, no 12, pp. 10335-10343 2009.

[20] E. I. Smotrova, T. M. Benson, J. Ctyroky, R. Sauleau, and A.I. Nosich, "Optical fields of the lowest modes in a uniformly active thin sub-wavelength spiral microcavity," Opt. Lett., vol. 34, no 24, pp. 3773-3775, 2009.

[21] J. Wiersig and M. Hentschel, "Combining directional light output and ultralow loss in deformed microdisks," Phys. Rev. Lett., vol. 100, pp. 033901, 2008.

[22] Q. H. Song, L. Ge, A.D. Stone, H. Cao, et al., "Directional laser emission from a wavelength-scale chaotic microcavity," Phys. Rev. Letts., vol. 105, pp. 103902, 2010.

[23] Q.H. Song, L. Ge, J. Wiersig, J.-B. Shim, J. Unterhinninghofen, A. Eberspacher, W. Fang, G. S. Solomon, and H. Cao, "Wavelength-scale deformed microdisk lasers," Phys. Rew. A., vol. 84, pp. 063843, 2011.

[24] E. I. Smotrova, V. O. Byelobrov, T. M. Benson, et al., "Optical theorem helps understand thresholds of lasing in microcavities with active regions," IEEE J. Quant. Electron., vol. 47, no 1, pp. 20-30, 2011.

[25] E. I. Smotrova, V. Tsvirkun, I. Gozhyk, et al., "Spectra, thresholds and modal fields of a kite-shaped microcavity laser," J. Opt. Soc. Am. B, vol. 40, no 6, pp. 1732-1742, 2013.

[26] J. H. Richmond, "Scattering by a dielectric cylinder of arbitrary crosssection shape," IEEE Trans. Antennas Propag., vol. 13, pp. 334-341, 1965. - Ibid. ., vol. 14, pp. 460-464, 1966.

[27] S.-K. Chang and K. K. Mei, "Application of the unimoment method to electromagnetic scattering of dielectric cylinders," IEEE Trans. Antennas Propag., vol. 24, no 1, pp. 35-42, 1976.

[28] N. Morita, "Surface integral representations for electromagnetic scattering from dielectric cylinders," IEEE Trans. Antennas Propag., vol. 26, pp. 261-266, 1978.

[29] K. Yashiro and S. Ohkawa, "Boundary element method for electromagnetic scattering from cylinders," IEEE Trans. Antennas Propag., vol. 33, no 4, pp. 383-389, 1985.

[30] S. V. Boriskina, T. M. Benson, P. Sewell, and A. I. Nosich, "Tuning of the 2D elliptic whispering-gallery-mode microdisk waveguide filters", IEEE/OSA J. Lightwave Technol., vol. 21, no 9, pp. 1987-1995, 2003.

[31] M. Lucido, G. Panariello, and F. Schettino, "Scattering by arbitrary polygonal cross-section dielectric cylinders at oblique incidence," IEEE Trans. Antennas Propag., vol. 58, no 2, pp. 540-550, 2010.

[32] A. F. Peterson, "The 'interior resonance' problem associated with surface integral equations of electromagnetics: numerical consequences and a survey of remedies," Electromagn., vol. 10, pp. 293-312, 1990.

[33] S. Amini and S. M. Kirkup, "Solutions of Helmholtz equation in the exterior domain by elementary boundary integral methods," $J$. Comput. Phys., vol. 118, pp. 208-221, 1995.

[34] C. Muller, Foundations of the Mathematical Theory of Electromagnetic Waves, Berlin, Springer, 1969 (German edition, 1957).

[35] V. Rokhlin, "Rapid solution of integral equations of scattering theory in 2-D," J. Comput. Phys., vol. 86, pp. 414-439, 1990.

[36] S. V. Boriskina, T. M. Benson, P. Sewell, and A. I. Nosich, "Optical modes in imperfect square and triangular microcavities," IEEE J. Quant. Electron., vol. 41, no 6, pp. 857-864, 2005.

[37] D. Colton, R. Kress, Inverse Acoustic and Electromagnetic Scattering Theory, Springer, Berlin (1998).

[38] L. Wang, J.A. Cox, and A. Friedman, "Modal analysis of homogeneous optical waveguides by the boundary integral formulation and Nystrom method," J. Opt. Soc. Am. A., 15 (1), 92-100 (1998).

[39] I. O. Sukharevsky, O. V. Shapoval, A. I. Nosich, and A. Altintas, "Validity and limitations of the median-line integral equation technique in the scattering by material strips of sub-wavelength thickness," IEEE Trans. Antennas Propag., vol. 62, no 7, pp. 3623-3631, 2014.

[40] M. Lebental, J. S. Lauret, R. Hierle, and J. Zyss, "Highly directional stadiumshaped polymer microlasers" Appl. Phys. Lett., vol. 88, pp. 031108, 2006.

[41] I. Gozhyk, G. Clavier, R. Méallet-Renault et al., "Polarization properties of solid-state organic lasers," Phys Rev A, vol. 86, no 4, pp. 043817, 2012. 